\title{
Enhancing Exploration with a Faceted Browser through Summarization
}

\author{
Grzegorz Drzadzewski \\ David R. Cheriton School of Computer Science \\ University of Waterloo \\ Waterloo, ON, N2L 3G1, Canada \\ gdrzadze@cs.uwaterloo.ca
}

\author{
Frank Wm. Tompa \\ David R. Cheriton School of Computer Science \\ University of Waterloo \\ Waterloo, ON, N2L 3G1, Canada \\ fwtompa@cs.uwaterloo.ca
}

\begin{abstract}
An enhanced faceted browsing system has been developed to support users' exploration of large multi-tagged document collections. It provides summary measures of document result sets at each step of navigation through a set of representative terms and a diverse set of documents. These summaries are derived from pre-materialized views that allow for quick calculation of centroids for various result sets. The utility and efficiency of the system is demonstrated on the New York Times Annotated Corpus.
\end{abstract}

\section{Categories and Subject Descriptors}

H.2.4 [Systems]: Textual databases; H.4.3 [Communications Applications]: Information browsers

\section{General Terms}

Algorithms, Design

\section{Keywords}

document repository, faceted browser, tags, result diversification, bursty terms

\section{INTRODUCTION}

Faceted browsing and search has emerged as a valuable technique for information access in many e-commerce sites, including Wal-Mart, Home Depot, eBay, and Amazon [15]. Faceted search has also been shown to be effective in helping users navigate document repositories, including the document collection at the University of North Carolina library, and it was preferred by users over traditional search interfaces based on text content alone [10].

With faceted browsing a user can navigate through a document collection by using facets as filters for specifying a document set of interest. Once the result set is narrowed down to a manageable size, the user can examine individual documents. Other than the count of items associated with

Permission to make digital or hard copies of part or all of this work for personal or classroom use is granted without fee provided that copies are not made or distributed for profit or commercial advantage and that copies bear this notice and the full citation on the first page. Copyrights for third-party components of this work must be honored. For all other uses, contact the Owner/Author. Copyright is held by the owner/author(s). DocEng'15, September 08-11, 2015, Lausanne, Switzerland. ACM 978-1-4503-3307-8/15/09.

DOI: http://dx.doi.org/10.1145/2682571.2797083. each metadata tag for each facet in the result set, faceted browsers do not usually provide any tools to help users understand the contents of the result set. Alternatively, systems such as Scatter/Gather display a link to the medoid document and the top- $k$ most frequently occurring terms [1], which gives a hint at the scope of each sub-collection. If the information provided is not particularly informative, the user must arbitrarily choose individual documents to examine in an attempt to understand what is in the isolated subcollection.

When navigating through many document sets, however, it is too time consuming to examine many documents from each set to determine whether the set as a whole or some subset may be of interest. As a user aid, document sets may be summarized to provide a quick overview of their contents. Common summaries for sets of documents include a representative set of terms $[7,8]$, a ranked list of representative documents $[1,2,5]$, and a natural language abstract [9]. Each of these approaches relies on being able to compute the centroid for any given document set.

We have developed an enhanced faceted browser interface that, in addition to letting a user perform traditional faceted browsing and searching, provides a user with summary information about the resulting document set. In our implementation, the summary includes a diverse set of representative documents and a well-chosen set of representative terms. In addition, the information scent [6] is enhanced by providing a set of representative terms for each of the important sub-collections of the result set (cf. multi-menus [11]). With these summary measures in place, a user is able to quickly grasp the topics covered by large result sets. This additional summary information can be especially useful in situations when the documents are not ordered in a favourable way (such as when using PubMed, where result sets are ordered by date rather than by relevance to the query).

We demonstrate the system on the New York Times (NYT) annotated corpus [12], which consists of 1.5 million tagged documents. The assigned tags are treated as facet values over which the user can search and browse.

\section{BASIC MEASURES OF INTEREST}

\subsection{Set Centroid}

Following standard practice in information retrieval, we model a document as a bag of terms represented by a document term vector (DTV), which is a vector of values where each entry corresponds to a term together with the term's (normalized) frequency in that document. A set of DTVs 
can be aggregated together to obtain a set centroid for the corresponding documents, which can be used by set summarization procedures. A set centroid $C$ is represented by a vector of term frequencies equal to the mean of all the DTVs for documents that belong to that set.

\subsection{Representative Terms}

Given a document set of interest $S_{c}$ and a superset $S_{p} \supseteq$ $S_{c}$ (its context), the burstiness of a term $w$ is measured using $B_{+}\left(w, C_{S_{c}}, C_{S_{p}}\right)$ as defined in Equation 1, where $C_{S_{c}}$ is the centroid of set $S_{c}$ and $C_{S_{p}}$ is the centroid of its context $S_{p}$ :

$$
B_{+}\left(w, C_{S_{c}}, C_{S_{p}}\right)=\frac{C_{S_{c}}[w] \cdot \text { freq }}{C_{S_{p}}[w] \cdot \text { freq }^{0.95}}
$$

A term is considered bursty if it has a sufficiently higher frequency of occurrence in set $S_{c}$ than its frequency of occurrence in the context set $S_{p}: B_{+}\left(w, C_{S_{c}}, C_{S_{p}}\right)>t$, where $t$ is pre-defined threshold (set to 1 in our system). The top $k$ or fewer (if fewer than $k$ terms are above the threshold) bursty terms found in $C_{S_{c}}$ constitute the set of representative terms $\mathbb{B}_{k}\left(S_{c}, S_{p}\right)$ for $S_{c}$ relative to $S_{p}$.

Since the absence of terms may often be as informative as their presence, we also want to show terms that occur significantly less in the set than in its superset. Therefore, we define negative burstiness, denoted $B_{-}\left(w, C_{S_{c}}, C_{S_{p}}\right)$, as defined in Equation 2.

$$
B_{-}\left(w, C_{S_{c}}, C_{S_{p}}\right)=\frac{C_{S_{p}}[w] \cdot f r e q}{C_{S_{c}}[w] \cdot f r e q^{0.95}}
$$

The top $k$ or fewer terms in $C_{S_{c}}$ with $B_{-}\left(w, C_{S_{c}}, C_{S_{p}}\right)>t$ are then displayed as part of the summary, representing a set of rare terms (denoted $\mathbb{B}_{k}^{-}\left(S_{c}, S_{p}\right)$ ) for $S_{c}$ relative to $S_{p}$.

As an example, Table 1 shows the set of positive and negative bursty terms (labelled "representative terms" and "rarely occurring terms," respectively) for the document set returned in response to the query "Music," along with the bursty terms associated with its two largest subsets: "Music AND Recordings (Audio)" and "Music AND Opera." The representative terms for the query "Music" are derived using $\mathbb{B}_{20}\left(S_{M u s i c}, S_{G}\right)$, where $S_{G}$ corresponds to all documents in the corpus, and those for "Music AND Recordings (Audio)" and "Music AND Opera" are defined with respect to "Music" using $\mathbb{B}_{20}\left(S_{\text {Music } \wedge \text { Recordings(Audio) }}, S_{\text {Music }}\right)$ for the former and $\mathbb{B}_{20}\left(S_{\text {Music }} \wedge\right.$ Opera,$\left.S_{\text {Music }}\right)$ for the latter; the rarely occurring terms use the corresponding negated formulae.

\section{REPRESENTATIVE DOCUMENTS}

\subsection{Approaches to Diversification}

There is a large body of research on search result diversification [14]. However, that work deals with diversification of results to ambiguous queries, for which different results are optimal depending on the interpretation of the query. That body of work is not directly applicable to our scenario since we do not deal with queries over text, but rather with navigation over tags. Because documents are assigned single instances of tags (we are not considering folksonomies, where the same tag can be assigned to a document by many users), documents matching a query over tags cannot be ordered based on the tag frequency in a similar fashion as term frequency is used to rank documents in a standard text search. Instead, in situations where documents are equal members
Table 1: Summary generated for the query "Music"

\begin{tabular}{|c|c|}
\hline Query & Music \\
\hline Doc. Count & 57,192 \\
\hline Rep. Doc. & Anniversaries Fill the Halls With Melody \\
\hline $\begin{array}{l}\text { Repres. } \\
\text { Terms }\end{array}$ & $\begin{array}{l}\text { sonata*,orchestra*,album*,concerto*, } \\
\text { beethoven*,guitar*,brahm*,quartet*, } \\
\text { philharmon*,melodi*,schubert*,mezzo*, } \\
\text { bassist*,melod*,saxophonist*,pianist*, } \\
\text { symphoni*,saxophon*,bariton*,cello* }\end{array}$ \\
\hline $\begin{array}{l}\text { Rarely } \\
\text { Occurring } \\
\text { Terms }\end{array}$ & report* \\
\hline Subquery & Music AND Recordings (Audio) \\
\hline Subset Count & 7,728 \\
\hline Rep. Doc. & A Once Proud Industry Fends Off Extinction \\
\hline $\begin{array}{l}\text { Repres. } \\
\text { Terms }\end{array}$ & 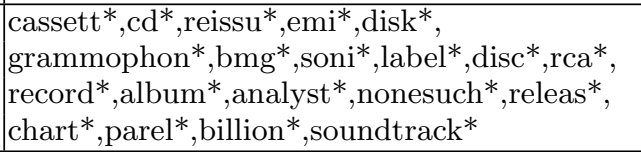 \\
\hline $\begin{array}{l}\text { Rarely } \\
\text { Occurring } \\
\text { Terms }\end{array}$ & $\begin{array}{l}\text { tulli*,pm*,averi*,intermiss*,tomorrow*, } \\
\text { ticket*,tonight*,fisher*,carnegi*,saturdai*, } \\
\text { hall*,alic*,costum*,metropolitan*,sundai* } \\
\text { recit*,auditorium*,lincoln*,festiv*,concert* }\end{array}$ \\
\hline Subquery & Music AND Opera \\
\hline Subset Count & 6,408 \\
\hline Rep. Doc. & Rossini, Hold the Pasta Sauce \\
\hline $\begin{array}{l}\text { Repres. } \\
\text { Terms }\end{array}$ & 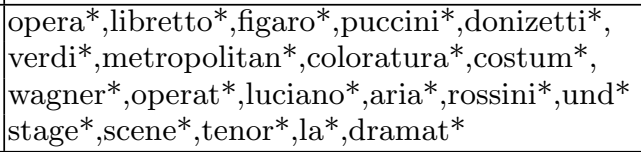 \\
\hline $\begin{array}{l}\text { Rarely } \\
\text { Occurring } \\
\text { Terms }\end{array}$ & $\begin{array}{l}\text { saxophonist*,bassist*,guitarist*,rapper*, } \\
\text { drummer*,guitar*,album*,,songwrit*,band*, } \\
\text { sonata*,jam*,rap*,punk*,drum*,jazz* } \\
\text { improvis*,funk*,hop*,saxophon*,rock* }\end{array}$ \\
\hline & \\
\hline
\end{tabular}
in the NYT.

of a set, representative documents are picked based on the centroid of the set, and the set is represented through a medoid [1] or through a set of documents that provide coverage of important concept terms stored in the centroid [2].

Using multiple documents to cover all the important concept terms found in the centroid of a set may be appropriate for representing a flat set, but in a collection with multitagged documents, as is the case with NYT, most sets can be partitioned into coherent subsets. For example, a set of documents defined by the "Music" tag can be partitioned into subsets, such as "Music AND Opera," derived by intersecting multiple dominant tags found in the set. These subsets impose a hierarchy over the set, which can be used for choosing a diverse representative set of documents, similarly to what was proposed by Vee et al. [16]. A set generated through this approach has documents that represent concepts found in many centroids of various subsets in the set of interest, and so will have a richer diversity than is possible with the coverage approach whose documents are optimized to cover a single centroid. This approach parallels the use of query reformulations to provide diverse sets of results for web searches [13]. 
Table 2: Analysis of documents returned for a query on bursty terms with respect to the corresponding tags (where only result sets of size greater than 100 are considered)

\begin{tabular}{|l|r|c|c|}
\hline $\begin{array}{c}\text { Query } \\
\text { Type }\end{array}$ & $\begin{array}{c}\text { Instance } \\
\text { Count }\end{array}$ & $\begin{array}{c}\text { Mean } \\
\text { Precision } \\
\text { at 100 }\end{array}$ & $\begin{array}{c}\text { Mean Rank } \\
\text { of Top Rele- } \\
\text { vant Doc. }\end{array}$ \\
\hline$t_{x}$ & 1,015 & $62.4_{\sigma=23.0}$ & $1.44_{\sigma=1.42}$ \\
$t_{x} \wedge t_{y}$ & 5,179 & $70.7_{\sigma=20.6}$ & $1.25_{\sigma=0.98}$ \\
\hline
\end{tabular}

Instead of calculating the medoid directly from the cosine distance of each document from the centroid, we take advantage of the infrastructure provided by a standard information retrieval system such as Lucene. Given a query, Lucene's ranking function ranks a document highly if the terms specified in a query appear frequently in the document and the document contains many of the terms specified in the query. This aligns well with the properties used for evaluating whether or not a document is a good representative of a set (i.e., it provides good coverage of the important concept terms of the centroid and those terms occur frequently in the document). As a result, the highest ranked document to a query consisting of the bursty terms of a set centroid makes a good representative document for the corresponding set. Since bursty terms are all relatively rare in the corpus, the ranking function will not likely have a strong bias towards documents that represent only some of the query terms.

We have examined the quality of this mapping between tags and the top 20 bursty terms describing the corresponding centroids (Table 2) to determine whether documents that contain the bursty terms tend to be in the expected subset (having the corresponding tag). The mapping was evaluated over all sets that have at least 100 documents and are defined by a single tag or by a conjunction of two tags. The top 20 bursty terms representing each of those sets were used to formulate queries. For each of those queries, a large proportion of the top 100 documents in the ranked lists of results are annotated with the corresponding tag or tags. Thus, there is a high correlation between the tags and the content text. In addition, the highest ranked result to the query on the bursty terms predominantly comes from the same set as the bursty terms. The existence of the required tags on many of the top 100 documents returned by a bursty terms query confirms the influence of the identified bursty terms in defining a document set. Furthermore, since many relevant documents are found in the top 100 results, various IR optimization techniques that take advantage of early termination can be used without fear of missing a representative document.

\subsection{Finding Representative Documents}

Given a query $Q$ defining a set of documents $S_{Q}$, let $t_{1}, t_{2}, \ldots, t_{n}$ be a ranked sequence of tags for which the rank of $\operatorname{tag} t_{i}$ is based on $\left|S_{Q \wedge t_{i}}\right|$ and the dissimilarity of $S_{Q \wedge t_{i}}$ with respect to both $S_{Q}$ and other subsets $S_{Q \wedge t_{j}}$ where $j<i$. (Details of the tag ranking algorithm are given in the full description of the system [3].) The $N+1$ diverse representative documents for $S_{Q}$, denoted by $\mathbb{D}_{Q}(N, k)$, are defined using Equation 4.

$$
\begin{gathered}
D_{Q}(i, k)= \begin{cases}R\left(\mathbb{B}_{k}\left(S_{Q}, S_{G}\right)\right) & \text { if } i=0 \\
R\left(\mathbb{B}_{k}\left(S_{Q \wedge t_{i}}, S_{Q}\right)\right) & \text { if } i>0\end{cases} \\
\mathbb{D}_{Q}(N, k)=\bigcup_{i=0}^{N} D_{Q}(i, k)
\end{gathered}
$$

where $S_{G}$ corresponds to all documents in the corpus and $R(x)$ returns the document in $S_{Q}$ that is top ranked when posing the text query using the terms in the set $x$.

For example, $D_{\text {Music }}(0,20)$ is found by taking the top 20 positive bursty terms, which include stemmed words such as sonata, orchestra, album, concerto, and beethoven, and issuing a text query with those terms. The highest ranked document in set $S_{\text {Music }}$ is chosen as a representative document, which happens to be the document with title $A n$ niversaries Fill the Halls With Melody as shown in Table 1. $D_{\text {Music }}(1,20)$ is found using the bursty terms in the largest subset, "Music $\wedge$ Recordings (Audio)," and $D_{\text {Music }}(2,20)$ uses the subset "Music $\wedge$ Opera." For our example, $\mathbb{D}_{\text {Music }}(2,20)$ is displayed in Table 1, and these three documents together form a diverse representative document set for $S_{\text {Music }}$.

\section{SYSTEM ARCHITECTURE}

The architecture of the faceted browsing system is shown in Figure 1. The system relies on the Partial Materialization Module, described by Drzadzewski and Tompa [4], which is central to efficient system performance. The module is designed to ensure fast access to requested centroids of document sets defined through Boolean operations over tags, while consuming minimal storage. Fast access to the centroid measure for document sets makes it feasible to produce the proposed summary measures for many sets within a short amount of time, so that a user can interactively explore large document collections. For example, with the help of the Partial Materialization Module, a centroid for a document set consisting of 142,000 documents can be obtained in under two seconds, whereas it would take 120 seconds to calculate the same centroid by aggregating individual documents.

When a user specifies a query over tags, the system produces a summary for the resulting documents by first accessing the Tag-Tag Index and a Tag-Document Index found in the Partial Materialization Module to produce a ranked sequence of $N$ tags, such that each chosen tag produces a large subset that is dissimilar from both the document set of interest and subsets produced by other selected tags. These tags are then individually combined with the original query as a conjunction in order to produce $N$ sub-queries. (For the query "Music," sub-queries such as "Music AND Recordings (Audio)" and "Music AND Opera" are generated.) The original query along with the $N$ sub-queries are then fed into the Partial Materialization Module, which produces a centroid for each of the queries along with the centroid for the whole corpus.

The resulting centroids are compared against each other to derive both positive and negative bursty terms (Eqns. 1 and 2) for the result set and principal subsets. The positive bursty terms are then used to form multiple text queries (a separate query for each set of bursty terms) (Eqns. 3 and 4). The highest ranked documents returned from each search 


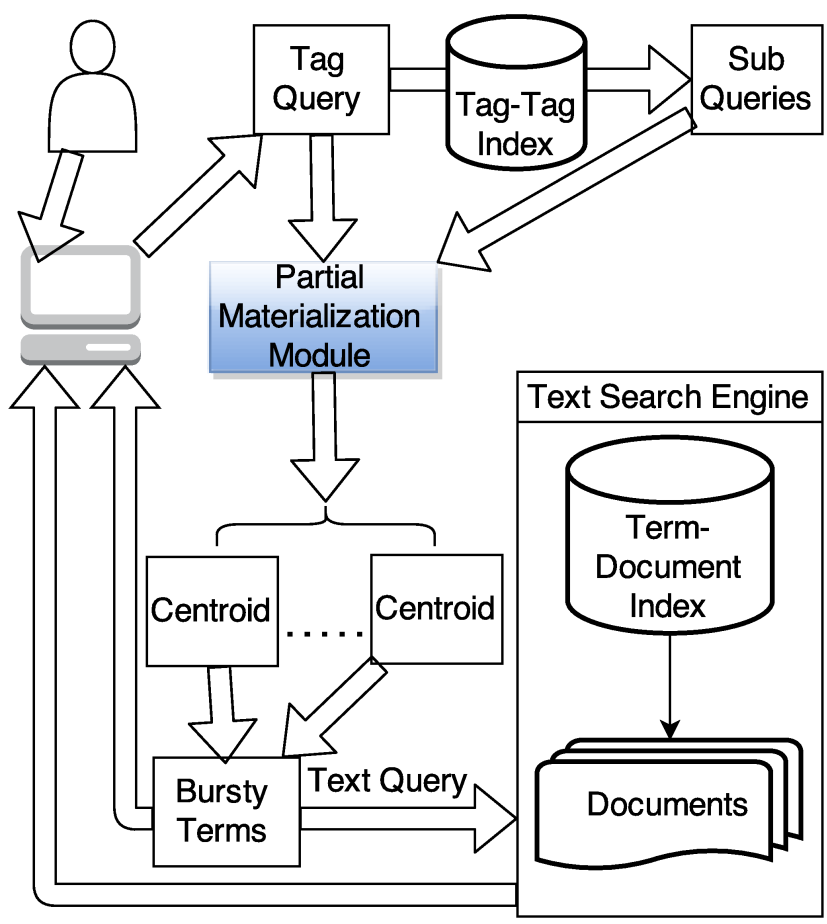

Figure 1: System for supporting faceted browsing

along with the corresponding bursty terms are returned to the user to produce the summary of the resulting set similar to Table 1.

The user interface is developed as a web app, and Lucene is used for indexing the documents and for supporting text queries through the standard ranking function. Additional information about the system is provided in the full system description [3] and on our website . $^{1}$.

\section{CONCLUSIONS}

Whereas a standard faceted browser in response to a user's query on a tag (e.g., "Music") would produce a list of all documents that have that tag (which happen to be 57,192 documents) as well as a list of facets and the number of documents associated with each facet ("Recordings (Audio)" with 7,728 documents, and "Opera" with 6,408 documents), our system also provides a set of diverse representative documents that come from the various subsets (defined by queries "Music AND Recordings (Audio)", and "Music AND Opera") as well as representative terms for the result set and each principal subset. This information provides a useful summary and an information scent so users can make informed decisions as to which subset(s) they may want to explore further.

\section{Acknowledgements}

Financial assistance from NSERC, Mitacs, and the University of Waterloo is greatly appreciated.

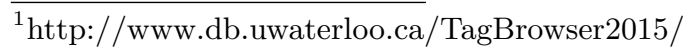

\section{REFERENCES}

[1] D. R. Cutting, J. O. Pedersen, D. R. Karger, and J. W. Tukey. Scatter/Gather: A cluster-based approach to browsing large document collections. In Proc. 15th Ann. Int. ACM SIGIR Conf. Res. Dev. Inf. Retr., pages 318-329, 1992.

[2] V. Deolalikar. Distance or coverage?: Retrieving knowledge-rich documents from enterprise text collections. In Proc. 23rd ACM Int. Conf. on Inf. Knowl. Manage., CIKM 2014, pages 1771-1774, 2014.

[3] G. Drzadzewski. On-Line Analytical Systems for Multi-Tagged Document Collections. PhD thesis, Cheriton Sch. Comp. Sci., Univ. Waterloo, 2015. in preparation.

[4] G. Drzadzewski and F. W. Tompa. Partial materialization for on-line analytical processing over multi-tagged document collections. Knowl. Inf. Syst., 2015. to appear.

[5] A. F. Gelbukh, M. Alexandrov, A. Bourek, and P. Makagonov. Selection of representative documents for clusters in a document collection. In 8th Int. Conf. Applic. Nat. Lang. Inf. Sys., pages 120-126, 2003.

[6] J. C. Goodwin, T. Cohen, and T. C. Rindflesch. Discovery by scent: Discovery browsing system based on the Information Foraging Theory. In 2012 IEEE Int. Conf. Bioinf. Biomed. Worksh., BIBMW 2012, pages 232-239, 2012.

[7] S. Goorha and L. Ungar. Discovery of significant emerging trends. In Proc. 16th ACM SIGKDD Int. Conf. Knowl. Disc. Data Min., KDD '10, pages 57-64, 2010.

[8] A. Popescul and L. H. Ungar. Automatic labeling of document clusters. unpublished, http://citeseer.nj. nec.com/popescul00automatic.html, 2000.

[9] D. R. Radev, H. Jing, M. Styś, and D. Tam. Centroid-based summarization of multiple documents. Inf. Process. Manage., 40(6):919-938, Nov. 2004.

[10] S. Ramdeen and B. M. Hemminger. A tale of two interfaces: How facets affect the library catalog search. J. Am. Soc. Inf. Sci. Technol., 63(4):702-715, 2012.

[11] D. R. Raymond. Personal data structuring in videotex. Technical Report CS-84-7, Cheriton Sch. Comp. Sci., Univ. Waterloo, February 1984.

[12] E. Sandhaus. The New York Times Annotated Corpus. Linguistic Data Consortium, Univ. Penn., http://www.ldc.upenn.edu/Catalog/catalogEntry.jsp? catalogId=LDC2008T19, 2008.

[13] R. L. T. Santos, C. Macdonald, and I. Ounis. Exploiting query reformulations for web search result diversification. In Proc. 19th Int. Conf. World Wide Web, WWW'10, pages 881-890, 2010.

[14] R. L. T. Santos, C. Macdonald, and I. Ounis. Search result diversification. Found. Trends Inf. Retr., 9(1):1-90, 2015.

[15] D. Tunkelang. Faceted Search. Synthesis Lectures on Information Concepts, Retrieval, and Services. Morgan \& Claypool Publishers, 2009.

[16] E. Vee, U. Srivastava, J. Shanmugasundaram, P. Bhat, and S. Yahia. Efficient computation of diverse query results. In Proc. 24th IEEE Int. Conf. Data Engin., ICDE 2008, pages 228-236, April 2008. 